\title{
Square Kilometre Array: the radio telescope of the XXI century
}

DOI:

$10.1134 / \mathrm{S} 1063772917040059$

\section{Document Version}

Accepted author manuscript

Link to publication record in Manchester Research Explorer

\section{Citation for published version (APA):}

Grainge, K., Alachkar, B., Amy, S., Barbosa , D., Bommineni, M., Boven, P., Braddock, R., Davis, J., Diwakar, P., Francis, V., Gabrielczyk, R., Gamatham, R., Garrington, S., Gibbon, T., Gozzard, D., Gregory, S., Guo, Y., Gupta, Y., Hammond, J., ... Wingfield, N. (2017). Square Kilometre Array: the radio telescope of the XXI century.

Astronomy Reports, 61(4). https://doi.org/10.1134/S1063772917040059

Published in:

Astronomy Reports

\section{Citing this paper}

Please note that where the full-text provided on Manchester Research Explorer is the Author Accepted Manuscript or Proof version this may differ from the final Published version. If citing, it is advised that you check and use the publisher's definitive version.

\section{General rights}

Copyright and moral rights for the publications made accessible in the Research Explorer are retained by the authors and/or other copyright owners and it is a condition of accessing publications that users recognise and abide by the legal requirements associated with these rights.

\section{Takedown policy}

If you believe that this document breaches copyright please refer to the University of Manchester's Takedown Procedures [http://man.ac.uk/04Y6Bo] or contact uml.scholarlycommunications@manchester.ac.uk providing relevant details, so we can investigate your claim.

\section{OPEN ACCESS}




\section{Square Kilometre Array: the radio telescope of the XXI century}

Keith Grainge ${ }^{1}$, Bassem Alachkar ${ }^{1}$, Shaun Amy $^{2}$, Domingos Barbosa ${ }^{3}$, Murali Bommineni ${ }^{4}$, Paul Boven ${ }^{5}$, Ralph Braddock ${ }^{1}$, John Davis ${ }^{6}$, Praveen Diwakar ${ }^{4}$, Vishal Francis ${ }^{7}$, Robert Gabrielczyk $^{1}$, Romeo Gamatham ${ }^{8}$, Simon Garrington ${ }^{1}$, Tim Gibbon ${ }^{9}$, David Gozzard ${ }^{10}$, Simon Gregory ${ }^{11}$, Yichen Guo ${ }^{12}$, Yashwant Gupta ${ }^{13}$, Jill Hammond ${ }^{1}$, David Hindley ${ }^{6}$, Uli Horn $^{14}$, Richard Hughes-Jones ${ }^{15}$, Michelle Hussey ${ }^{1}$, Samantha Lloyd ${ }^{1}$, Siju Mammen ${ }^{16}$, Simeon Miteff ${ }^{14}$, Vivek Mohile ${ }^{4}$, Jaco Muller ${ }^{1}$, Swami Natarajan ${ }^{16}$, John Nicholls ${ }^{17}$, Richard Oberland $^{1}$, Mike Pearson ${ }^{1}$, Tim Rayner ${ }^{17}$, Sascha Schediwy ${ }^{10}$, Richard Schilizzi ${ }^{1}$, Supriya Sharma $^{4}$, Simon Stobie ${ }^{10}$, Mark Tearle ${ }^{1}$, Bo Wang ${ }^{12}$, Bruce Wallace ${ }^{8}$, Lijun Wang ${ }^{12}$, Rajesh Warange $^{4}$, Richard Whitaker ${ }^{1}$, Althea Wilkinson ${ }^{1}$, Neil Wingfield ${ }^{1}$

${ }^{1}$ Jodrell Bank Centre for Astrophysics, Alan Turing Building, School of Physics \& Astronomy, The University of Manchester, Oxford Road, Manchester M13 9PL, UK.

${ }^{2}$ CSIRO Astronomy and Space Science, Australia Telescope National Facility,PO Box 76, Epping, NSW 1710, Australia.

${ }^{3}$ Grupo de Radio Astronomia, Basic Sciences \& Enabling Technologies, Instituto de Telecomunicações, Campus Universitario de Santiago, 3810-193 Aveiro, Portugal.

${ }^{4}$ Persistent Systems Limited, 9A/12, Karve Road, Bharati Niwas Colony, Erandwane, Pune, Maharashtra 411004, India.

${ }^{5}$ Joint Institute for VLBI ERIC (JIVE), Oude Hoogeveensedijk 4, 7991 PD Dwingeloo, The Netherlands.

${ }^{6}$ National Physical Laboratory, Hampton Road, Teddington, Middlesex TW11 OLW, UK.

${ }^{7}$ STFC, Rutherford Appleton Laboratory, Harwell Campus, OX11 0QX, UK.

${ }^{8}$ SKA South Africa, $3^{\text {rd }}$ Floor, The Park, Park Road, Pinelands 7405, South Africa.

${ }^{9}$ Nelson Mandela Metropolitan University, Department of Physics, Building 13, Summerstrand Campus (South), University Way, Port Elizabeth 6031, South Africa.

${ }^{10}$ School of Physics, The University of Western Australia, 35 Stirling Highway, Perth WA 6009, Australia.

${ }^{11}$ STFC, Daresbury Laboratory, Sci-Tech Daresbury, Warrington, Cheshire, WA4 4AD, UK.

${ }^{12}$ Tsinghua University, Joint Institute for Measurement Science, The state key lab of precision Measurement Technology and Instrument, Department of Precision Instruments, Beijing 100084, China.

${ }^{13}$ National Centre for Radio Astrophysics, Tata Institute of Fundamental Research, Pune University Campus, Post Bag 3, Ganeshkhin, PO Pune 411 007, Maharashtra, India.

${ }^{14}$ South African National Research Network, CSIR Meraka Institute, Building 43 Block D, Meiring Naude Road, Scientia, Pretoria 0001, South Africa.

${ }^{15}$ GEANT, City House, 126-130 Hills Road, Cambridge CB2 1PQ, UK.

${ }^{16}$ Tata Consultancy Services, TCS House, Raveline Street, 21 DS Marg, Fort Mumbia, Maharashtra 400001, India.

${ }^{17}$ AARNet, Building 1, Binary Centre, 3 Richardson Place, North Ryde NSW 2113, Australia.

\section{Abstract}

The Square Kilometre Array (SKA) will be the world's largest and most sensitive radio telescope. It will address fundamental unanswered questions about our Universe including how the first stars and galaxies formed after the big bang, how dark energy is accelerating the expansion of the Universe, the role of magnetism in the cosmos, the nature of gravity, and the search for life beyond Earth. This project envisages the construction of 133 15-m antennas in South Africa and 131,072 log-periodic antennas in Australia, together with the associated infrastructure in the two desert sites. In addition, the SKA is an exemplar Big Data project, with data rates of over 10 Tbps being transported from the telescope to HPC/HTC facilities.

\section{Introduction}

The Square Kilometre Array (SKA) is an international project to build a next generation radio telescope. The telescope will be built in two phases, with even the Phase 1 instrument having a survey speed around 100 times faster than the current state-of-the-art. The SKA will be the world's largest astronomical instrument and will join such instruments as ALMA, JWST and E-ELT as one of the world's great observatories. It will be the next transformational step in radio astronomy, and will shape its future for many decades. The detailed design for Phase 1 
of the telescope is currently underway, with Preliminary Design Reviews passed by all the telescope elements and Critical Design Review on schedule for late 2017. Construction of the SKA will start in 2018 and it will be built over a 5 year period by international industry within a cost cap set in 2013 of $€ 650 \mathrm{M}$.

The SKA comprises two separate interferometric arrays on two different continents. The SKA-low telescope will operate between frequencies of $50-350 \mathrm{MHz}$ and will be deployed at the Murchison Radio Astronomy Observatory, Australia. It will incorporate a total of 131,072 log-periodic antennas, which are grouped into 512 stations of 256 antennas each. 296 of these stations will be configured into a central core area, with the remaining 216 deployed along three spiral arms to give a maximum baseline of $65 \mathrm{~km}$. The SKA-mid telescope will operate between frequencies of $0.35-13.8 \mathrm{GHz}$ and will be located in the Karoo desert in South Africa. It will be made up of 133 offset Gregorian antennas with 15m diameter, and will additionally incorporate the 64 antennas of 13.5m diameter being built for the MeerKAT telescope. Again, there will be a central core of antennas and three spiral arms allowing a maximum baseline of $150 \mathrm{~km}$.

In this paper we will briefly discuss the key science drivers for the SKA and one of the dominant challenges for this project, the vast amount of data that will be produced, transported and processed. We will then focus into one of the design areas required for tackling this exemplar Big Data problem, namely that of the networking required for the SKA.

\section{Scope of SKA Science}

The originating ideas for the SKA arose in the 1980s and were then developed in the 1990s. Central to these ideas was the goal of imaging neutral hydrogen through its redshifted 1.4 $\mathrm{GHz}$ emission line and it was realised that the sensitivity required to image typical galaxies out to redshift 2 at better than arcsecond resolution would require a telescope with a collecting area of around $1 \mathrm{~km}^{2}$ and baselines of around $100 \mathrm{~km}$. While neutral hydrogen imaging is still one of the key drivers for the telescope, the science goals of the SKA are now much broader. The SKA science case is captured in "Advancing Astrophysics with the Square Kilometre Array” (Braun et al., 2015) and contains 135 chapters written by 1,213 contributors. The book spans many diverse areas of astrophysics, with transformational discoveries possible in the following areas:

- Strong-field Tests of Gravity with Pulsars and Black Holes (Kramer \& Stappers 2015). The surveying capabilities of the SKA will be sufficient to increase the number of normal and milli-second pulsars by more than 10-fold. Through extremely accurate timing measurements of these pulsars, the SKA will be able to search for gravitational wave by effectively using the whole of our galaxy as a detector. In addition, pulsar systems are exquisite laboratories for studying physics in very strong gravitational fields and will allow testing of the predictions of General Relativity.

- Cosmic Dawn and the Epoch of Reionization (Koopmans et al. 2015). The first stars in the Universe were born around 100 million years after the Big Bang. Very little is known about this era of Cosmic Dawn, and the most promising avenue for exploration comes through observations of highly redshifted neutral hydrogen. These first stars and AGN began to heat and then ionise the matter in the Universe during the Epoch of Reionization (EoR). Measurements of neutral hydrogen will allow us to directly image these EoR structures. 
- The Transient Radio Sky (Fender et al. 2015). In recent years a new phenomenon of Fast Radio Bursts (FRBs) has been discovered. These FRBs are believed to be due to extremely violent explosions in galaxies at cosmological distances. FRBs are interesting in their own right, but also offer the possibility of allowing detection of the missing baryons in the Universe. Monitoring the behaviour of other transient objects will allow astronomers to study the disruption of stars by super-massive black holes.

- Cosmology \& Dark Energy (Maartens et al. 2015). A number of techniques will allow the SKA to investigate cosmology, including: strong and weak gravitational lensing; neutral hydrogen intensity mapping; radio galaxy surveys out to high redshift; and HI galaxy redshift surveys. Through these we hope to learn about the nature of Dark Energy, modified gravity, and primordial non-Gaussianity.

- The Origin and Evolution of Cosmic Magnetism (Johnston-Hollitt et al. 2015). Radio measurements of Faraday rotation offer a unique method for exploring magnetic fields. These are known to be of vital importance in galaxy formation and to the evolution of galaxy clusters. In addition we will also be able to explore the origin of cosmic magnetic fields.

- Galaxy Evolution probed by Neutral Hydrogen (Staveley-Smith \& Oosterloo 2015). The SKA will have the sensitivity to allow the imaging of substantial number of high-redshift galaxies in $\mathrm{HI}$ at resolutions of $<1$ arcsec for the first time, vastly improving our understanding of the formation, growth and evolution of galaxies.

- The Cradle of Life \& Astrobiology (Hoare et al. 2015). One of the key unresolved issues in the understanding of planetary formation is the mechanism by which small pebbles in the disk round a young star are able to coalesce to form boulders, which then go on to form planets. The SKA's wavelength range is well matched to that of these pebbles and so it will be able to observe this phase of planetary evolution.

- Galaxy and Cluster Evolution probed in the Radio Continuum (Prandoni \& Seymour). The process of star formation reached a peak of activity about 3 billion years after the Big Bang and since then the rate has dropped. The mode of star formation at this epoch seems to have a different character to that at the present day and further study will require high sensitivity radio continuum observations allowed by the SKA in order to be able to penetrate the obscuring matter round these sites.

\section{Big Data and the SKA}

The volumes of data that the SKA will generate are vast. Each of the 197 antennas of the SKA-mid will generate around 90 Gbits/s of digitised payload data, giving a total of 17.7 Tbit/s to be transported from the geographically distributed antennas back to a Central Processing Facility (CPF). Here the data are processed, with interferometric visibilities being formed by correlation of the signals between antenna pairs and in addition a search being formed for pulsar candidates. The processing required by these steps is around 50 Pflops and the hardware and firmware is being designed by the Central Signal Processor (CSP) consortium. These intermediate data are then passed to the Science Data Processor (SDP) for further analysis, requiring around 250 Pflops of additional compute.

For the SKA-low the volumes are even larger. The signals from each of the log-periodic antennas are transmitted on RF-over-fibre to a bunker where each polarisation is Nyquist sampled at 8 bits, generating around 1,490 Tbit/s. The data are beamformed and then, in a similar fashion to SKA-mid, passed to first CSP and then SDP for processing.

These data volumes and processing requirements make the SKA an exemplar Big Data challenge. The design of the CSP and SDP are beyond the scope of this paper, and in the next 
section we concentrate on the challenges posed by the data transport requirements of the SKA.

\section{Networking for the SKA}

The SKA Signal and Data Transport (SADT) Consortium is comprised of 15 different institutes from 8 countries, and is led by the University of Manchester. The SADT Consortium has been allocated responsibility by the SKA Organisation for design of the various networks that connect the other elements of the telescope.

\subsection{Overview of SKA networking}

The scope of the Signal and Data Transport (SADT) element is to provide 3 logically distinct networks for both of the telescopes that comprise the SKA.

1) The astronomical data network. This is itself split into 3 sub-systems which have very different requirements:

a) The Digital Data Backhaul (DDBH) network transports signals from the receptors to the CSP.

b) The CSP-SDP network transports data products from the CSP to the SDP.

c) The SDP network distributes data from the SDP to the regional SKA Regional Data Centres.

2) The Synchronisation and Timing (SAT) network. This provides frequency and clock signals from a central clock ensemble to all elements of the system to maintain phase information to the required accuracy for all receptors, and timing signals for data identification and time critical activities at the receptors, the CSP and SDP.

3) The Non-Science Data Network (NSDN). This connects all locations of the telescope system and transports the monitoring and control information that is the responsibility of the Telescope Manager (TM) element, and the general communications traffic.

The Network Manager (NMGR) sub-system provides the local monitoring and management of the DDBH and CSP-SDP science data networks, the NSDN and the associated networking equipment. Local monitoring and control for the SAT is provided separately by the SAT.LMC sub-system. SADT is also responsible for the local infrastructure (LINFRA) requirements specific to SADT and outside the scope of the INFRA consortium, for example optical fibre reticulation design and SADT repeater shelter buildings.

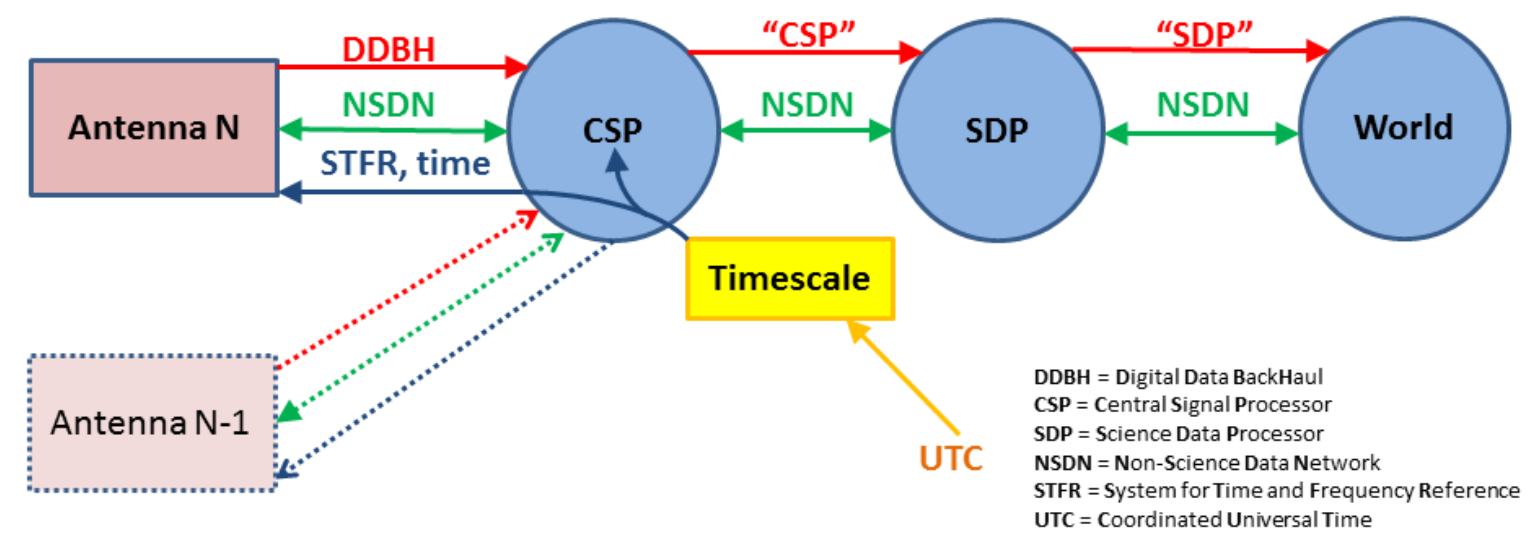

Figure 1 Schematic of the SADT networks. The three astronomical data networks are shown in red and the data flows are unidirectional. The network that distributes time, frequency and phase is shown in blue. The non-science data network is shown in green. An instance of the above diagram will be required for each of the two telescopes. In the case of SKA-low the DDBH and Station Time and Frequency Reference (STFR) networks will only be provided to the outer stations/antennas as part of the SADT Element. 
The scope of the SADT networking connectivity is summarised in Figure 1. In the following sections we give some more details about the various SADT sub-systems appropriate to a snapshot in time corresponding to the Preliminary Design Review (PDR).

\subsection{Astronomical Data Transport Networks}

\subsubsection{DDBH Network}

The Digital Data Back-Haul (DDBH) network will be a fully managed, self-contained, COTS solution with short reach Ethernet client interfaces that are completely vendor agnostic. From a cost driven point of view, it will use simple layer 1 or layer 2 switching technologies. Wherever possible the same technology has been used for both telescopes allowing for greater efficiencies in operational costs across the SKA1 deployment. The equipment will support a rich feature-set of management, monitoring and remote access protocols. Figure 2 shows the logical network diagrams for the two telescopes.

\subsubsection{SKA-mid telescope}

For the SKA-mid telescope, there will be two different implementations of the DDBH system dependent on the fibre distance of the antenna from the Central Processing Facility (CPF) building. There are approximately 95 inner array dishes that fall within a distance of less than $10 \mathrm{~km}$. These will have 'pizza box' equipment deployed at the Dish sites and present a single 100G short reach optical client interface compliant with the IEEE 802.3 Ethernet standard, 100GBASE-SR4. At the CSP side of the link the proposal is to aggregate the 95 dishes into a larger chassis based equipment using standard LR-4 transceivers on the line side and presenting 100GBASE-SR4 short reach interfaces to the CSP client. The remaining 38 outer array dishes $(>10 \mathrm{~km})$ will use long reach DWDM transmission equipment, but with the same 100GBASE-SR4 client interface.
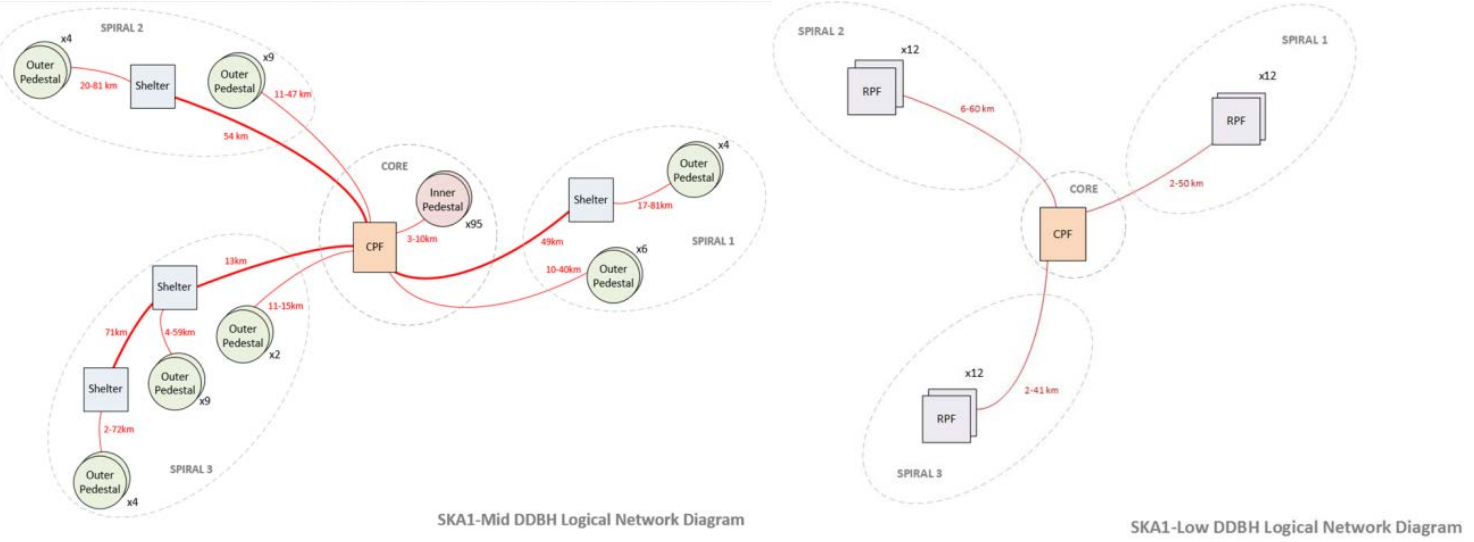

Figure 2 The logical network diagrams for the DDBH network transmitting telescope data from the receptors back to the Central Processing Facility (CPF) for the SKA-mid (left) and SKA-low (right) telescopes.

\subsubsection{SKA1-low telescope}

For the SKA1-LOW telescope, the DDBH network needs to support a client data capacity of 40GE from each of the stations outside the core area. There are 36 SKA-low clusters of 6 stations each located in Remote Processing Facilities (RPFs) less than 80km from the CPF building. The optical client interface in the COTS design will comply with IEEE 802.3 Ethernet standards with 6x40GBASE-SR4 capacity per RPF location. The line side interface 
will be provided by optical DWDM equipment using coherent modulation formats. All switches will be non-blocking to maintain transparency.

\subsubsection{CSP-SDP Network}

The SKA CSP-SDP Network provides long distance high bandwidth connectivity between the Central Signal Processor (CSP) locations, and the Science Data Processor (SDP) complex for each of the telescopes. It will carry the science data traffic from the CSP to SDP facilities as well as non-science data such as monitoring and control from TM and other auxiliary site traffic. These services will be separated at the optical layer. Figure 3 shows a schematic of the CSP-SDP connecting the CSP, the SDP and the Engineering Operations Centre (EOC) locations.

Due to the data rates and distances required, 7.4 Tbit/s over a distance of $\sim 910 \mathrm{~km}$ for the observatory in South Africa and $5.8 \mathrm{Tbit} / \mathrm{s}$ over a distance of $~ 820 \mathrm{~km}$ for the observatory in Australia, the equipment choice is constrained to standard long-haul coherent transmission equipment used by telecommunications carriers. Based on the current road maps, the line or transmission side technology will use a encoding technique similar to Dual Polarisation Quadrature Phase-Shift Keying modulation (DP-QPSK) with Coherent Detection (CD) and Dense Wavelength Division Multiplexing (DWDM. No regeneration is required over these distances, but optical amplification will be needed approximately every 70 to $100 \mathrm{~km}$. COTS equipment and fibre will be used and will support 120 channels each running at 200Gbps. Ethernet with support for Jumbo frames that carry an IP MTU 9000 bytes will be used.

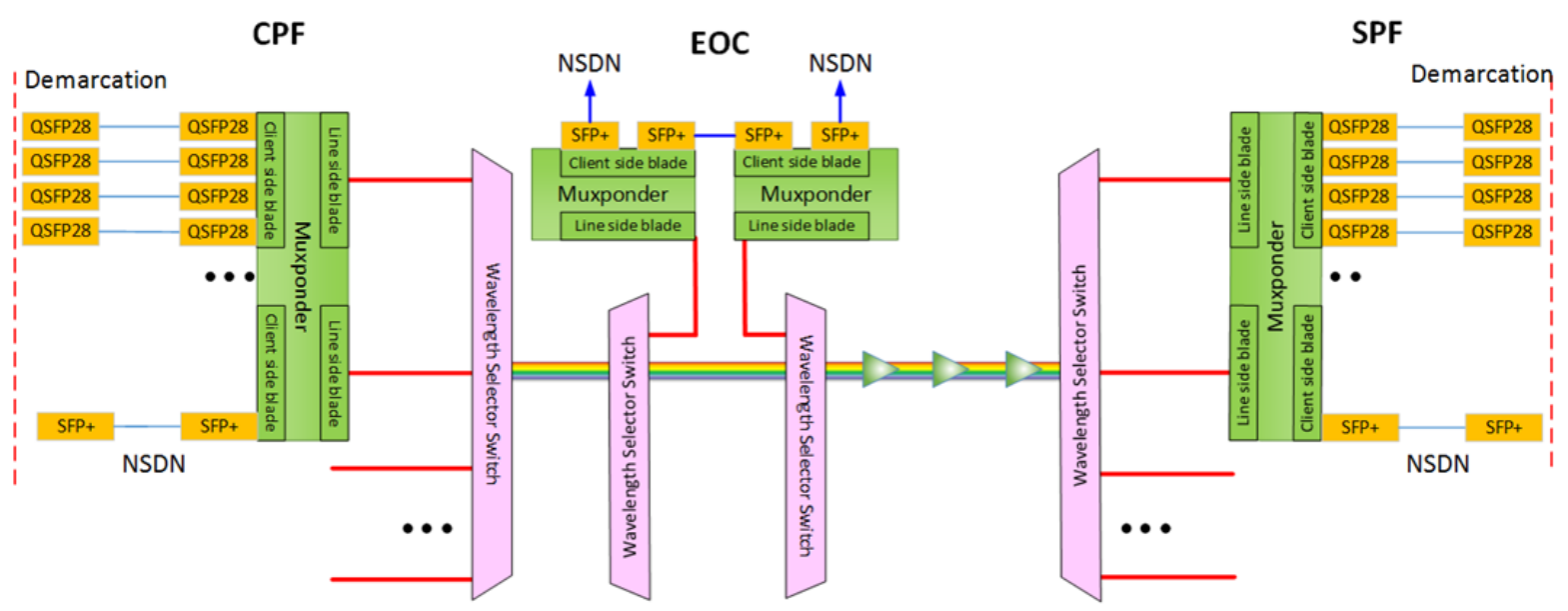

Figure 3 Schematic for the CSP-SDP network

\subsection{Synchronisation and Timing}

There are multiple different, but related, requirements for Synchronisation and Timing for the SKA:

1. Ensuring that the whole synthesis array is phase coherent. A maximum permitted coherence loss of $2 \%$ equates to 0.2 radians of phase error, which, at a maximum observing frequency of 20 $\mathrm{GHz}$, corresponds to accuracies of $\sim 1 \mathrm{ps}$.

2. Providing high precision long-term timing, for astrophysical phenomena such as pulsars and transients. In particular, pulsar monitoring experiments require timing accuracies of $10 \mathrm{~ns}$ over time periods of 10 years. 
3. Providing absolute time for system management, antenna pointing, beam steering, time stamping of data and producing regular timing ticks.

4. Providing frequency standards for Local Oscillators (LOs), digitiser clocks, low frequency square waves or Walsh functions for phase switches and noise diode.

5. For VLBI operations, in which some, or all, of the phased SKA core will be combined with other telescopes around the world, the requirements of (1) apply, possibly with some degree of relaxation relative to the other, independent telescopes.

A block diagram of how these requirements are met by the SKA Synchronisation and Timing element is shown in Figure 4 and its sub-systems are discussed below.

\subsubsection{SAT Timescale}

The design of the reference timescales for the SKA telescopes assumes that near-identical timescales will be constructed for each of the Mid telescope in South Africa and for the Low telescope in Australia. The timescales will use Coordinated Universal Time (UTC) as their time and frequency reference. A robust design is proposed for each timescale, based on the use of an ensemble of three active hydrogen masers as the reference clocks. The first two of these masers each produces a physical realisation of the SKA Timescale. One of these will act as the master realisation and the other as back-up. Physical switching (triggered by the appropriate software command) between master and back-up timescale should enable continued operation following individual hardware failures and enable the SKA Timescales to meet their operational requirements. The third maser allows for a "Three Cornered Hat" arrangement to allow identification of which of the two timescale realisations is the source of an anomaly should they disagree.

A key requirement of the SKA Timescales is an accurate knowledge of their time offsets with respect to UTC. This is required for pulsar timing and other applications. Global Navigation Satellite System (GNSS) time transfer will be used as the primary time transfer method. Each SKA Timescale will operate three GNSS receivers, which will be regularly calibrated using portable calibrating GNSS receivers. Direct traceability to UTC will be achieved by the SKA Timescales becoming UTC $(\mathrm{k})$ facilities, and the time transfers being computed by the Bureau International des Poids et Mesures (BIPM).

A steering mechanism is required to keep the time and frequency of the SKA Timescale close

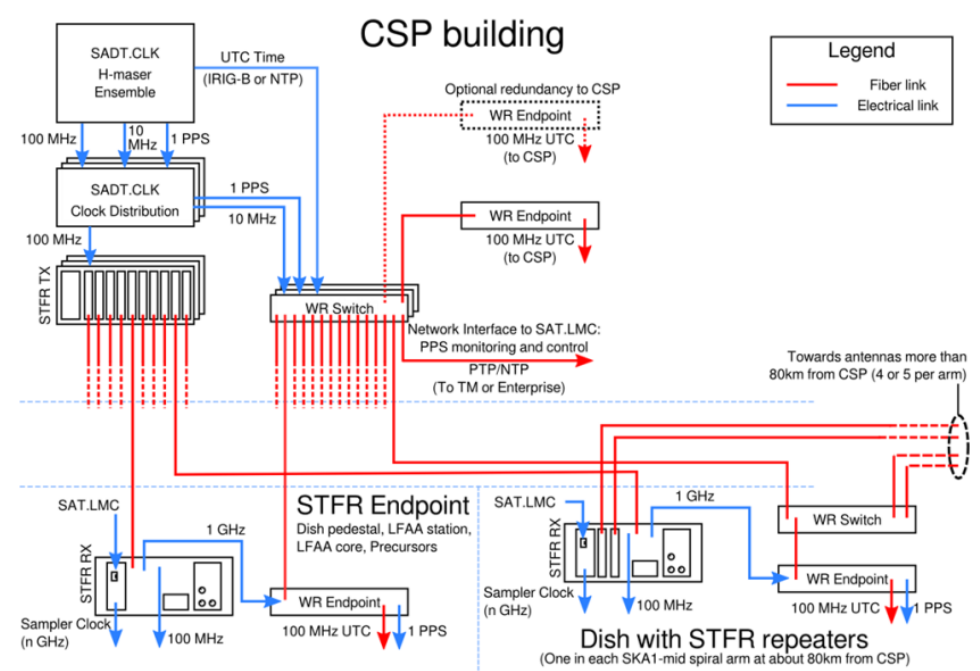

Figure 4 Block diagram of the time and frequency reference distribution system to that of UTC. Timescale signals are generated from the outputs of the active hydrogen masers, which are then adjusted by applying small frequency changes. This process both enables the resulting SKA master and back-up timescales to be aligned to UTC and leaves the free running active hydrogen masers unperturbed. The steering process may be achieved using either manual or automatically generated frequency steers. A 
steering algorithm will be provided to offer the possibility of automating the SKA Timescale steering process. In all cases it will be ensured that the magnitude of the steers will be below the stochastic noise level to avoid perturbing the underlying timescale stability and astronomical timing measurements.

Monitoring the performance of the SKA Timescales is key to their successful operation. Automatic measurements and analysis of many of the components of the SKA Timescales will be included in the design, along with anomaly detection. This process will be supported by operator observations which may be undertaken on a weekly basis. An annual programme of calibration, performance evaluation and servicing will form part of the operations of the SKA Timescales.

\subsubsection{System for Timing and Frequency Reference}

The SAT.STFR (Synchronisation and Timing - Station Time and Frequency Reference) carries out the function of delivering standard frequency references and timing signals derived from the SKA frequency and timescale reference to the receptors and other locations. The reference signals need to be delivered with sufficient precision to enable each of the telescopes to be phase-coherent, and to support the demanding requirements on absolute time for scientific observations such as pulsar timing on timescales of decades.

These signals will be transported by optical fibre. Environmental influences, such as temperature changes or mechanical vibrations, affect the optical path length of the optical fibre links, thereby degrading the stability of the delivered reference signals. The designed solutions all measure this effect by sending a signal out and back again over the same fibre. As the signal travels both ways over the same fibre, the actual delay or phase variation at the destination will be half of the measured value and can be compensated.

For the distribution of the reference frequency, we have two designs, one concept developed by Tsinghua University, and the other by the University of Western Australia. Both systems deliver not only a reference frequency, but include a means of synthesizing all the required frequencies for driving samplers at the receptors, where required.

The UTC time reference makes use of a publically available hardware and software designs from a collaborative project called 'White Rabbit' which delivers sub-ns accuracy using mostly COTS components and sub-systems.

\subsubsection{SAT Local Monitoring and Control}

The SAT.LMC will provide monitor and control functionality to the Clocks and STFR subsystems of the SAT system. The SAT.LMC primary role will be to receive monitor data from all of the SAT sub-systems and relay this information on to the overall SKA Telescope Manager (TM). In addition the SAT.LMC will provide fault diagnostic support and provide alarm management support to the TM.

\subsection{Non-Science Data Network}

The Non-Science Data Network (NSDN) consists of an access layer, distribution switches and IP/MPLS Core routers, with a presence across the entire observatory. It is designed as a converged network to carry multiple services that support the operation of the telescopes. It is primarily responsible for transporting monitoring and control information between the 
Telescope Manager (TM) and the Local Monitoring and Control (LMC) interfaces of all subsystems, but it also transports general data communication services such as internet connectivity, voice and infrastructure services.

The NSDN architecture is highly resilient and scalable, and consists of a core network, a distribution layer (where required) and an access layer. The NSDN will be deployed at the CPF and observatory campus locations such as accommodation and support facilities, the receptor pedestals or repeater shelters, the Science Processing Facility (SPF), the Engineering Operations Centre (EOC) and the Science Operations Centre (SOC). This network will either interface to, or incorporate, the current precursor site networks to ensure a holistic network approach.

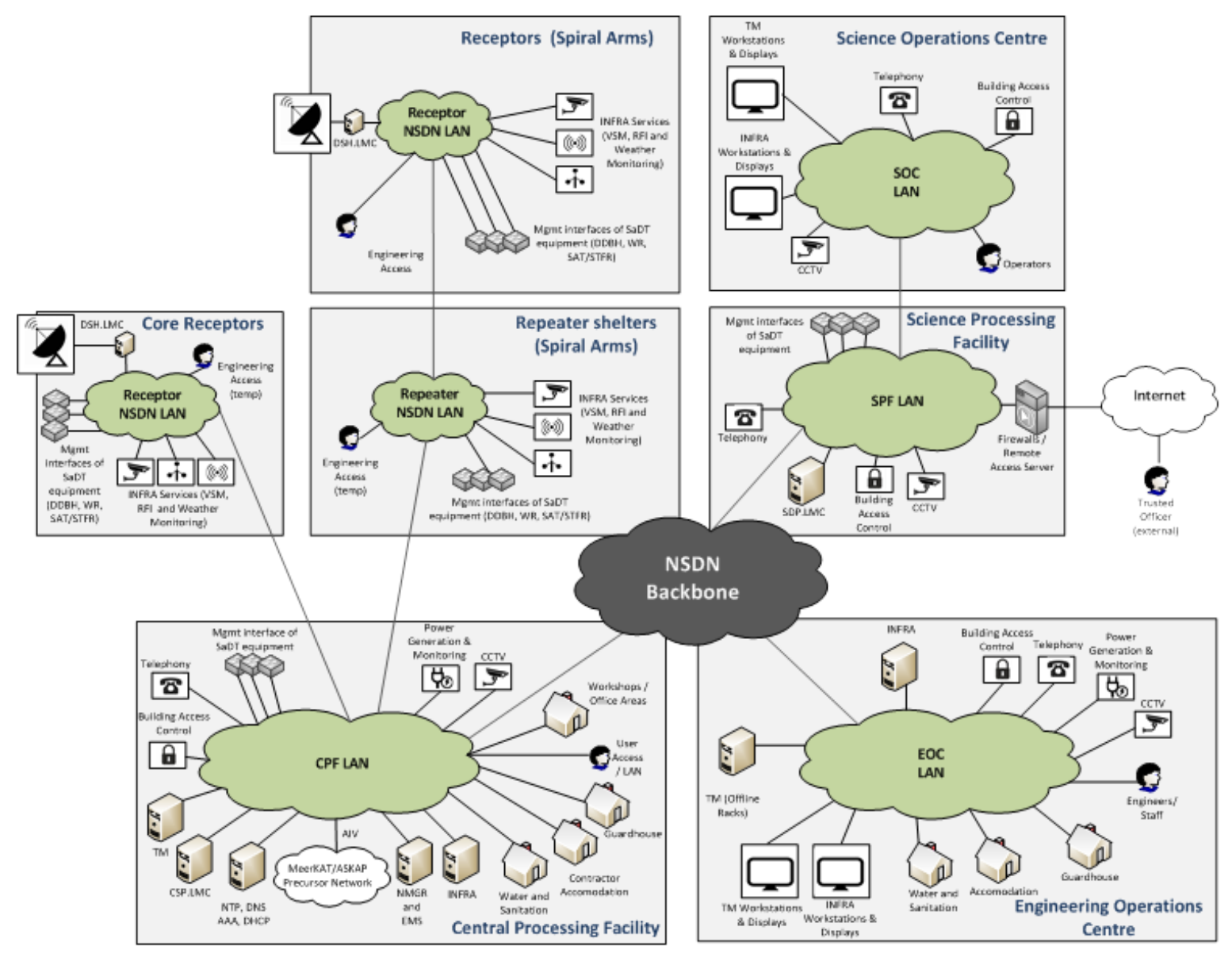

Figure 5 Spatial distribution of locations and services carried by NSDN

The NSDN is designed to support security requirements as they are developed. NSDN will allow the segregation of services and the flexibility to integrate security appliances such as firewalls at core locations. In addition, connectivity of trusted offices and by trusted officers is implicit in the design.

\subsection{Network Manager}

The SADT.NMGR sub element is responsible for monitoring the DDBH, NSDN, CSP-SDP networks and the associated infrastructure. The SADT.NMGR element comprises a Network Management System (NMS), and interacts with external systems, particularly the Telescope 
Manager. The NMS may consist of a single COTS solution or a combination of COTS systems from multiple vendors.

The NMS will be presented to the users in the form of a dashboard that gives a single view of the overall network health and availability, as well as generating alarms and sending alerts in case of any errors detected. Users will be able to zero-in on specific portions of the network topology to analyse and pinpoint the causes of any deviations from normal operation of the network.

\subsection{Network Architecture}

As described above, the SADT system consists of several different networks. The simplest approach for the architecture of these systems would be to have separate systems without any overlap. However, to obtain the lowest possible OPEX and CAPEX costs, the hardware in these subsystems needs to be shared as much as possible. To determine the overall SADT architecture separate networks are assessed with respect to their functionalities and requirements. On the basis of this assessment a single architecture for the four systems is determined.

\subsection{Local Infrastructure}

The fibre optic network is the physical layer across which all of the SKA1 telescope networks operate. The design and successful implementation of the fibre network is paramount to the successful operation of the SKA1 telescopes, providing both flexibility to accommodate changing requirements as network technologies develop and additional requirements identified. The challenge is to achieve these results in the most technically acceptable, economic manner to achieve the maximum utilisation and life expectancy of the fibre network installation.

To this end the fibre network will use standards-based, single mode fibre types in multi-core cables as commonly used by the major telecommunication equipment manufacturers. The cable must be installed under controlled conditions and expert supervision, utilising installation technologies that complement the natural terrain conditions, to ensure that lifetime expectancies of the fibre network are met with minimal maintenance and/or repair requirements during the operational period of the telescope. The fibre network will be optimised in the future in conjunction with the power and road network development to share trenches wherever possible, and to align with the infrastructure construction schedules to benefit from these activities wherever possible and to minimise duplication of effort and disruption to both parties in order to contain costs.

\section{Summary}

The SKA telescope will be the largest scientific project on the Earth. The range of science that the SKA will explore is vast and it offers to make truly transformational discoveries in the fields of understanding of the earliest stages of the Universe, gravitational waves, General Relativity and clues as to the origins of life. With a design operational lifespan of 50 years, the SKA will be the world's leading radio observatory for much of the $21^{\text {st }}$ century. Its construction will be beyond the capabilities of universities and research institutes and will require the industrialisation of radio astronomy. Resolving the Big Data challenges posed by the SKA will generate and develop novel solutions that will have impact beyond radio 
astronomy. One of these challenges is provision of the networks to carry the huge volumes of data that will be generated. These networks are the backbone of the telescope, without which the telescope is inoperable and unable to produce the scientific results for which it has been developed.

\section{Acknowledgements}

This paper describes work being carried out for the SKA Signal and Data Transport (SaDT) consortium as part of the Square Kilometre Array (SKA) project. The SKA project is an international effort to build the world's largest radio telescope, led by SKA Organisation with the support of 10 member countries.

\section{Bibliography}

Braun, R., et al., 2015, “Advancing Astrophysics with the Square Kilometre Array”, in Advancing Astrophysics with a Square Kilometre Array, PoS(AASKA14)174

Fender, R. et al. 2015, “The Transient Universe with the Square Kilometre Array”, in Advancing Astrophysics with a Square Kilometre Array, PoS(AASKA14)051

Hoare, M. et al. 2015, "SKA and the Cradle of Life", in Advancing Astrophysics with a Square Kilometre Array, PoS(AASKA14)115

Johnston-Hollitt, M. et al. 2015, "Using SKA Rotation Measures to Reveal the Mysteries of the Magnetised Universe”, in Advancing Astrophysics with a Square Kilometre Array, PoS (AASKA14)092

Koopmans, L. et al. 2015, "The Cosmic Dawn and Epoch of Reionisation with SKA", in Advancing Astrophysics with a Square Kilometre Array, PoS(AASKA14)001

Kramer, M. \& Stappers, B., 2015, “An overview of pulsar science with the SKA”, in Advancing Astrophysics with a Square Kilometre Array, PoS(AASKA14)036

Maartens, R. et al. 2015, "Overview of Cosmology with the SKA", in Advancing Astrophysics with a Square Kilometre Array, PoS(AASKA14)016

Prandori, I. \& Seymour, N., 2015, "Revealing the Physics and Evolution of Galaxies and Galaxy Clusters with SKA Continuum Surveys”, in Advancing Astrophysics with a Square Kilometre Array, PoS(AASKA14)067

Staveley-Smith, L. \& Oosterloo, T., 2015, “HI Science with the Square Kilometre Array”, in Advancing Astrophysics with a Square Kilometre Array, PoS(AASKA14)092 